INPLASY

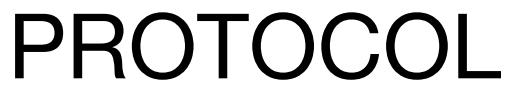

To cite: Zhang et al. Effects of arsenic trioxide combined with platinum drugs in treatment of cervical cancer:A protocol for systematic review and metaanalysis of randomized controlled trials. Inplasy protocol 202080130. doi: 10.37766/inplasy2020.8.0130

Received: 31 August 2020

Published: 31 August 2020

Corresponding author:

DaoCheng Li

daochenli@126.com

Author Affiliation:

The First Affiliated Hospital of Guangzhou University of Chinese Medicine

Support: Administration of TCM of GD.

Review Stage at time of this submission: The review has not yet started.

Conflicts of interest: None.

\section{Effects of arsenic trioxide combined with platinum drugs in treatment of cervical cancer: A protocol for systematic review and meta-analysis of randomized controlled trials}

Zhang, YW'; Pan, D2; Yang, HS3; Li, Z4; Huang, JX5; Li, DC6; He, ZY7; Li, $\mathrm{H}^{8}$.

Review question / Objective: Population: Patients with cervical cancer Intervention: Radical hysterectomy for cervical cancer after two courses of chemotherapy (AS2O3 combined with platinum drugs) Comparison: Radical hysterectomy for cervical cancer after two courses of chemotherapy (TP chemotherapy regimen Outcome: Clinical total effective rate and adverse reactions ;Cervical cancer tumor markers (SCCAg/CYFRA21-1), quality of life and immune function. Study design: Randomized, double-blind trials.

Condition being studied: The literatures of AS2O3 combined with platinum drugs related to cervical cancer have shown inconsistent results, and there is currently no high quality of systematic review to evaluate the effects of AS2O3 combined with platinum drugs in cervical cancer patients; team members include professors, doctoral students, and master students, all of whom are proficient and independently searching literature.

INPLASY registration number: This protocol was registered with the International Platform of Registered Systematic Review and Meta-Analysis Protocols (INPLASY) on 31 August 2020 and was last updated on 31 August 2020 (registration number INPLASY202080130).

\section{INTRODUCTION}

Review question / Objective: Population: Patients with cervical cancer Intervention: Radical hysterectomy for cervical cancer after two courses of chemotherapy (AS2O3 combined with platinum drugs)
Comparison: Radical hysterectomy for cervical cancer after two courses of chemotherapy (TP chemotherapy regimen Outcome: Clinical total effective rate and adverse reactions ;Cervical cancer tumor markers (SCCAg/CYFRA21-1), quality of life 
and immune function. Study design: Randomized, double-blind trials.

Condition being studied: The literatures of AS2O3 combined with platinum drugs related to cervical cancer have shown inconsistent results, and there is currently no high quality of systematic review to evaluate the effects of AS2O3 combined with platinum drugs in cervical cancer patients; team members include professors, doctoral students, and master students, all of whom are proficient and independently searching literature.

\section{METHODS}

Search strategy: English and Chinese literature about AS2O3 combined with platinum drugs treatment for cervical cancer published before August 31, 2020 will be systematic searched in PubMed, Embase, Web of Science, Cochrane Library, Open Grey, Clinicaltrials.gov, Chinese Clinical Trial Registry, WANFANG, VIP Chinese Science and Technology Journal Database, CNKI, Chinese biomedical document service system(SinoMed).

Participant or population: Patients with cervical cancer Intervention.

Intervention: Radical hysterectomy for cervical cancer after two courses of chemotherapy (AS2O3 combined with platinum drugs).

Comparator: Radical hysterectomy for cervical cancer after two courses of chemotherapy (TP chemotherapy regimen.

Study designs to be included: Randomized, double-blind trials.

Eligibility criteria: Patients with cervical cancer diagnosed pathologically; The staging standard refers to FIGO in 2008: IB2, IIA, IIB; 20-60 years old; Blood analysis before treatment, normal liver and kidney function; No evidence of distant metastasis; No serious heart, liver, kidney and blood system and other important organ diseases; The patient has signed an informed consent form.
Information sources: PubMed, Embase, Web of Science, Cochrane Library, Open Grey, Clinicaltrials.gov, Chinese Clinical Trial Registry, WANFANG, VIP Chinese Science and Technology Journal Database, CNKI, Chinese biomedical document service system(SinoMed).

Main outcome(s): Clinical total effective rate and adverse reactions.

Additional outcome(s): Cervical cancer tumor markers (SCCAg/CYFRA21-1), quality of life and immune function.

Quality assessment / Risk of bias analysis: The risk of bias assessment chart of inclusion studies will be produced by using Review Manager 5.3 software.

Strategy of data synthesis: Meta-analysis will be produced by Review Manager 5.3 and be presented as forest plot.

Subgroup analysis: Subgroup analysis will be performed according to age, ethnicity, FIGO 2008 stage and Histological grade and type of cervical cancer.

Sensibility analysis: We will use sensitivity analysis to test the stability and reliability of meta-analysis. It will be conducted by 2 methods: eliminating each study one by one; using random-effect model (DerSimonian \& Laird method) to test the results after using the fixed effect model.

Language: English and Chinese literature.

Country(ies) involved: China.

Keywords: cervical cancer, meta-analysis, Arsenic Trioxide (AS203), protocol, systematic review.

Contributions of each author:

Author 1 - YaWen Zhang.

Author 2 - Di Pan.

Author 3 - HaiShi Yang.

Author 4 - Zige Li.

Author 5 - JiaXin Huang.

Author 6 - DaoCheng Li.

Author 7 - ZeYang He.

Author 8 - Haiying Li. 